\title{
Semiactive Vibration Control for Horizontal Axis Washing Machine
}

\author{
Barış Can Yalçın and Haluk Erol \\ Mechanical Engineering Faculty, Istanbul Technical University, Gümüşsuyu, 34439 Istanbul, Turkey \\ Correspondence should be addressed to Haluk Erol; erolha@itu.edu.tr
}

Received 23 February 2015; Revised 1 July 2015; Accepted 27 July 2015

Academic Editor: Evgeny Petrov

Copyright ( 2015 B. C. Yalçın and H. Erol. This is an open access article distributed under the Creative Commons Attribution License, which permits unrestricted use, distribution, and reproduction in any medium, provided the original work is properly cited.

\begin{abstract}
A semiactive vibration control method is developed to cope with the dynamic stability problem of a horizontal axis washing machine. This method is based on adjusting the maximum force values produced by the semiactive suspension elements considering a washing machine's vibration data (three axis angular position and three axis angular acceleration values in time). Before actuation signals are received by the step motors of the friction dampers, vibration data are evaluated, and then, the step motors start to narrow or expand the radius of bracelets located on the dampers. This changes the damping properties of the damper in the suspension system, and thus, the semiactive suspension system absorbs unwanted vibrations and contributes to the dynamic stability of the washing machine. To evaluate the vibration data, the angular position and angular acceleration values in three axes are defined in a function, and the maximum forces produced by semiactive suspension elements are calculated according to the gradient of this function. The relation between the dynamic stability and the walking stability is also investigated. A motion (gyroscope and accelerometer) sensor is installed on the top-front panel of the washing machine because a mathematical model of a horizontal axis washing machine suggests that the walking behavior starts around this location under some assumptions, and therefore, calculating the vibrations occurring there is crucial. Semiactive damping elements are located under the left and right sides of the tub. The proposed method is tested during the spinning cycle of washing machine operation, increasing gradually from $200 \mathrm{rpm}$ to $900 \mathrm{rpm}$, which produces the most challenging vibration patterns for dynamic stability. Moreover, the sound power levels produced by the washing machine are measured to evaluate the noise performance of the washing machine while the semiactive suspension system is controlled. The effectiveness of the proposed control method is shown through experimental results.
\end{abstract}

\section{Introduction}

During the operation of a washing machine, the main dynamic problem is an unbalanced load of unpredictable position and magnitude. As the drum rotates, clothes separate from each other, causing stability problems. A washing machine is dynamically stable if unbalanced load only produces the forces and torques in such a way as to create a small oscillation about the equilibrium point of washing machine. However, even for these small oscillation values, a washing machine may show walking instability behaviour because of washing machine's rigidness; thus, it can be said that just because a washing machine is dynamically stable does not mean that it has walking stability as well. Conrad and Soedel investigated the main reasons for walking instability in horizontal and vertical axis washing machines using rudimentary dynamic models [1]. However, the washing machine model that they used did not contain elastic components, and therefore, the effects of passive suspension elements were neglected. Conrad later studied a more advanced dynamic mathematical model of horizontal and vertical axis washing machines for selected constraints and showed an approach to implementing dynamic elements such as a spring and a damper into the static model of such washing machines [2]. These studies proved the importance of suspension elements. This led to the question of what the effect of semiactive suspension systems that have adjustable spring and damping coefficients would be if spring and damping elements having constant coefficients can contribute to the dynamic stability of washing machines. Researchers thus began focusing on 
solving the dynamic stability problem of washing machines using semiactive suspension systems. Semiactive suspension elements can successfully deal with the dynamic stability problem by manipulating the centrifugal forces acting on the cabinet by storing and dissipating them so as to safely transfer the centrifugal forces produced by clothes from the drum to the ground. Changing the magnitude and direction of transferred forces contributes to the stable dynamic behavior of washing machines [3]. However, the question is how much of the force should be stored and how much should be dissipated. In this regard, the selection of the type of semiactive suspension is crucial. Magnetorheological (MR) dampers appear to be the best choice because their damping coefficients can be changed in wide variety; furthermore, they show fast response and are easy to control through an electronic interface [4]. However, using them in the home appliance industry may be uneconomical owing to production constraints. Nevertheless, several studies have focused on using them for reducing vibrations in a semiactive suspension system, that is, for dynamic stability, and for noise reduction. Spelta et al. [5] improved an adaptive control method to deal with unwanted vibrations and noise using a semiactive suspension system (MR-controllable friction damper); this algorithm is based on changing the damping parameters in response to the vibrations.

In our study, the working structure of the semiactive suspension system is based on adjusting the maximum force produced under load. Step motors are installed on suspension elements located under the tub. For each actuation signal, the step motors narrow or expand bracelets on suspension elements made of polyurethane; therefore, the elastic properties of polyurethane start changing, and the stiffness and damping coefficients change accordingly. However, owing to the structure of the suspension system, the only known parameter is the maximum force that can be produced by suspension elements. In the manufacturing process of the suspension system, the maximum reaction forces under load for each elastic level of the suspension system are measured. No motion sensor is installed on the suspension system, and the actual stiffness and actual damping coefficients are unknown; therefore, the actual reaction force of the suspension system cannot be determined. Using this type of suspension system provides an economic advantage compared with MR semiactive suspensions; on the other hand, the actual stiffness and actual damping values of suspension elements are unknown during operation.

Some other studies have investigated vibration reduction using classic suspension systems for horizontal washing machines. Türkay et al. investigated parametric optimization methods for reducing the maximum orbit displacement. In their method, grid and sequential quadratic programming optimization methods were used [6]. In another study, they used the Newton-Euler method to derive a nonlinear time-variant rigid body dynamic model of a classic suspension system of a horizontal axis washing machine for experimental assessment [7]. Boyraz and Gündüz investigated the optimization of the vibration characteristics using a generic algorithm [8]. Öztürk and Erol optimized the dynamic behavior of a horizontal axis washing machine using
Adams (MSC), a commercial multibody simulation software [9].

Papadopoulas and Papadimitriou provided a mathematical model of a horizontal axis washing machine assuming that the machine is a rigid structure [10]. In this model, the maximum angular velocity that can be reached (walking stability threshold) is determined according to other parameters such as the weight of clothes and friction between the ground and the feet. In our study, this mathematical model was first adapted to a washing machine with a semiactive suspension system; therefore, the assumption of rigidness is no longer completely valid, and the stiffness and damping coefficients can be considered in the equation for the maximum angular velocity that can be reached. This situation provides an opportunity for manipulation and proves that if the forces produced by semiactive suspension elements are changed appropriately, the maximum angular velocity can reach higher levels. However, the lack of modeling of some elastic components of the washing machine (cabinet, tub, etc.) and the assumption that the friction coefficient between the ground and the feet of the washing machine is constant may be weaknesses of the mathematical model. Sound performance of the washing machine must also be considered while the vibration performance is being improved [11]. Therefore, sound power outputs of the washing machine for the proposed control method are measured during operation and compared with those while the washing machine is working with an uncontrolled suspension system. The similarities and differences of the outputs at different stages of the spin cycle are discussed.

Our study aims to solve the dynamic stability problem using low-cost equipment (suspension system that works based on adjusting the maximum force produced, motion sensor, and data acquisition card), and the success of the control method for walking instability is evaluated according to two criteria: Papadopoulas and Papadimitriou's walking stability threshold adapted to a horizontal axis washing machine working with a semiactive suspension system and reduction of the arithmetic mean of the position and acceleration values in three axes.

\section{Theory}

An unbalanced load owing to rotating clothes in the drum has unpredictable position and magnitude values. To simplify the calculations, some assumptions have to be made. The first assumption is that the radius of clothes in the drum, $r_{d}$, is constant. $N_{y}$ and $N_{z}$ are the components of the $y$ - and $z$-axes of the unbalanced force produced by rotating clothes in the drum:

$$
\begin{aligned}
& N_{z}=m_{d} \omega^{2} r_{d} \cos \alpha+m_{d} g, \\
& N_{y}=m_{d} \omega^{2} r_{d} \sin \alpha,
\end{aligned}
$$

where $m_{d}$ is the mass of the unbalanced load; $g$ is the gravitational acceleration; $\alpha$ is the angle between the load and the $y$-axis; and $\omega$ is the angular velocity of the drum around the $x$-axis. 


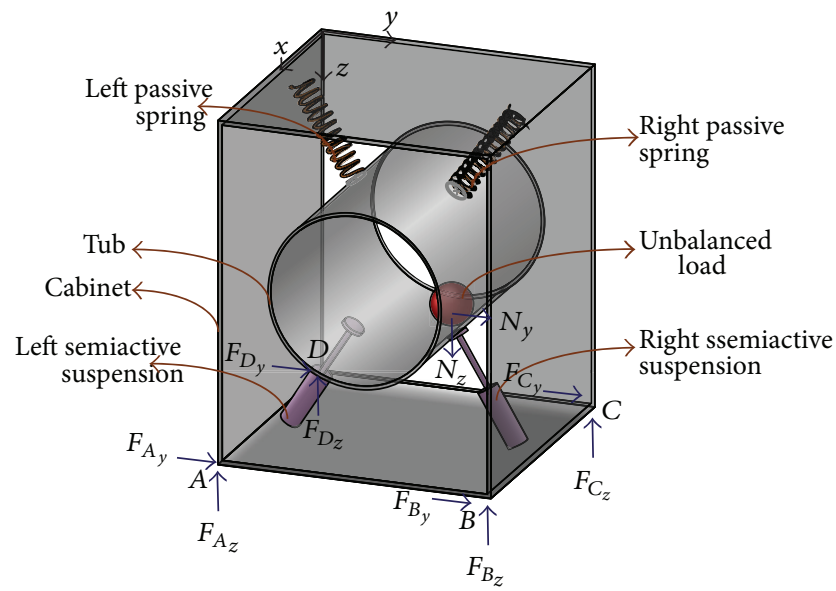

FIGURE 1: Forces acting on washing machine.

The second assumption pertains to the rigidness of the washing machine, with the forces produced by rotating clothes $\left(N_{y}, N_{z}\right)$ and friction forces on feet $\left(F_{A}, F_{B}, F_{C}\right.$, and $F_{D}$ ) shown in Figure 1.

Points $A, B, C$, and $D$, respectively, indicate the front left, front right, back left, and back right feet of the washing machine.

If it is assumed that the washing machine has no suspension, the following moment of force equations are used to derive walking stability threshold. In the equations, moment of force parameters are calculated with respect to $y$-axis shown in Figure 1:

$$
\begin{aligned}
& F_{A_{z}}+F_{B_{z}}=\frac{M_{c} g x_{1}}{d}+\frac{N_{z} x_{2}}{d}, \\
& F_{C_{z}}+F_{D_{z}}=\frac{M_{c} g\left(d-x_{1}\right)}{d}+\frac{N_{z}\left(d-x_{2}\right)}{d}, \\
& F_{A_{y}}+F_{B_{y}}=\frac{N_{y} x_{2}}{d} \\
& F_{C_{y}}+F_{D_{y}}=\frac{N_{y}\left(d-x_{2}\right)}{d}
\end{aligned}
$$

where $d$ is the distance between the front and the back feet; $x_{1}$ is the distance between the back feet and the center of gravity of the washing machine; $x_{2}$ is the distance between the back feet and the center of gravity of the load; and $M_{c}$ is the mass of the washing machine.

When $x_{2}$ is greater than half of $d$, the sum of $F_{A}$ and $F_{B}$ on the $y$-axis becomes greater than that of $F_{C}$ and $F_{D}$ on the $y$-axis:

$$
\begin{aligned}
F_{A_{y}}+F_{B_{y}} & >F_{C_{y}}+F_{D_{y}}, \\
x_{2} & >\frac{d}{2} .
\end{aligned}
$$

When $x_{1}$ is less than half of $d$, the sum of $F_{C}$ and $F_{D}$ on the $z$-axis becomes greater than that of $F_{A}$ and $F_{B}$ on the $z$-axis:

$$
\begin{aligned}
F_{C_{z}}+F_{D_{z}} & >F_{A_{z}}+F_{B_{z}}, \\
x_{1} & <\frac{d}{2} .
\end{aligned}
$$

Considering these conditions, walking instability first occurs around the front feet because they are subjected to more force than the back feet. Thus, in our study, a motion sensor is installed on the middle of the front-top panel of the washing machine.

$n$ is the friction between the ground and the feet:

$$
n\left(F_{A_{z}}+F_{B_{z}}\right)=F_{A_{y}}+F_{B_{y}} .
$$

Using (3) and (5), this equation can be rewritten as

$$
n\left(\frac{M_{c} g x_{1}}{d}+\frac{N_{z} x_{2}}{d}\right)=\frac{N_{y} x_{2}}{d} .
$$

Substituting (1) and (2) into (10) gives

$$
\begin{aligned}
& n\left(\frac{M_{c} g x_{1}}{d}+\frac{\left(m_{d} \omega^{2} r_{d} \cos \alpha+m_{d} g\right) x_{2}}{d}\right) \\
& =\frac{\left(m_{d} \omega^{2} r_{d} \sin \alpha\right) x_{2}}{d}, \\
& \omega=\sqrt{\frac{n g\left(M_{c} x_{1}+m_{d} x_{2}\right)}{m_{d} r_{d} x_{2}(\sin \alpha-n \cos \alpha)} .}
\end{aligned}
$$

To make the equation independent of $\alpha$, the derivative of (12) with respect to $\alpha$ has to be equal to 0 :

$$
\alpha=\tan ^{-1}\left(-\frac{1}{n}\right) .
$$

Then, $\omega$ becomes

$$
\omega=\sqrt{\frac{n g\left(M_{c}\left(x_{1} / x_{2}\right)+m_{d}\right)}{m_{d} r_{d} \sqrt{\left(1+n^{2}\right)}} .}
$$

All equations shown above hold under the assumption of the rigidness of the washing machine, and they change when the washing machine contains a suspension system. Because of the structure (spring and damping elements) of the semiactive suspension system and the passive suspension system, $N_{z}$ and $N_{y}$ given by (1) and (2) change, and their new forms are, respectively, given by

$$
\begin{aligned}
N_{z}= & m_{d} \omega^{2} r_{d} \cos \alpha+m_{d} g-k_{1} \widehat{p}_{1} \sin \beta_{1} \\
& -k_{2} \widehat{p}_{2} \sin \beta_{2}-b_{1} \frac{d \widehat{p}_{1}}{d t} \sin \beta_{1}-b_{2} \frac{d \widehat{p}_{2}}{d t} \sin \beta_{2} \\
& -k_{3} \widehat{p}_{3} \sin \beta_{3}-k_{4} \widehat{p}_{4} \sin \beta_{4}, \\
N_{y}= & m_{d} \omega^{2} r_{d} \sin \alpha-k_{1} \widehat{p}_{1} \cos \beta_{1}-k_{2} \widehat{p}_{2} \cos \beta_{2} \\
& -b_{1} \frac{d \widehat{p}_{1}}{d t} \cos \beta_{1}-b_{2} \frac{d \widehat{p}_{2}}{d t} \cos \beta_{2} \\
& -k_{3} \widehat{p}_{3} \cos \beta_{3}-k_{4} \widehat{p}_{4} \cos \beta_{4},
\end{aligned}
$$


where $k_{1}$ is the stiffness coefficient of the left semiactive suspension element; $k_{2}$ is the stiffness coefficient of the right semiactive suspension element; $k_{3}$ is the stiffness coefficient of the left passive spring; $k_{4}$ is the stiffness coefficient of the right passive spring; $b_{1}$ is the damping coefficient of the left semiactive suspension element; $b_{2}$ is the damping coefficient of the right semiactive suspension element; $\beta_{1}$ is the angle between the ground and the left semiactive suspension element; $\beta_{2}$ is the angle between the ground and the right semiactive suspension element; $\widehat{p}_{1}$ is the displacement of the left semiactive suspension element; $\widehat{p}_{2}$ is the displacement of the right semiactive suspension element; $\beta_{3}$ is the angle between the top panel and the left passive spring; $\beta_{4}$ is the angle between the top panel and the right passive spring; $\widehat{p}_{3}$ is the displacement of the left passive spring; and $\widehat{p}_{4}$ is the displacement of the right passive spring.

Substituting these new forms into (3) and (5) gives

$$
\begin{aligned}
& F_{A_{z}}+F_{B_{z}}=\frac{M_{c} g x_{1}}{d} \\
& +\frac{\left(m_{d} \omega^{2} r_{d} \cos \alpha+m_{d} g-k_{1} \widehat{p}_{1} \sin \beta_{1}-k_{2} \widehat{p}_{2} \sin \beta_{2}-b_{1}\left(d \widehat{p}_{1} / d t\right) \sin \beta_{1}-b_{2}\left(d \widehat{p}_{2} / d t\right) \sin \beta_{2}-k_{3} \widehat{p}_{3} \sin \beta_{3}-k_{4} \widehat{p}_{4} \sin \beta_{4}\right) x_{2}}{d}, \\
& F_{A_{y}}+F_{B_{y}} \\
& =\frac{\left(m_{d} \omega^{2} r_{d} \sin \alpha-k_{1} \widehat{p}_{1} \cos \beta_{1}-k_{2} \widehat{p}_{2} \cos \beta_{2}-b_{1}\left(d \widehat{p}_{1} / d t\right) \cos \beta_{1}-b_{2}\left(d \widehat{p}_{2} / d t\right) \cos \beta_{2}-k_{3} \widehat{p}_{3} \cos \beta_{3}-k_{4} \widehat{p}_{4} \cos \beta_{4}\right) x_{2}}{d}, \\
& n\left[M_{c} g x_{1}\right. \\
& \left.+(m_{d} \omega^{2} r_{d} \cos \alpha+m_{d} g \overbrace{-k_{1} \widehat{p}_{1} \sin \beta_{1}-k_{2} \widehat{p}_{2} \sin \beta_{2}-b_{1} \frac{d \widehat{p}_{1}}{d t} \sin \beta_{1}-b_{2} \frac{d \widehat{p}_{2}}{d t} \sin \beta_{2}-k_{3} \widehat{p}_{3} \sin \beta_{3}-k_{4} \widehat{p}_{4} \sin \beta_{4}}^{A}) x_{2}\right) \\
& =(m_{d} \omega^{2} r_{d} \sin \alpha \overbrace{-k_{1} \widehat{p}_{1} \cos \beta_{1}-k_{2} \widehat{p}_{2} \cos \beta_{2}-b_{1} \frac{d \widehat{p}_{1}}{d t} \cos \beta_{1}-b_{2} \frac{d \widehat{p}_{2}}{d t} \cos \beta_{2}-k_{3} \widehat{p}_{3} \cos \beta_{3}-k_{4} \widehat{p}_{4} \cos \beta_{4}}^{B}) x_{2} .
\end{aligned}
$$

Thus, the walking stability threshold changes as follows:

$$
\omega=\sqrt{\frac{n\left(M_{c} g x_{1}+m_{d} g x_{2}+A x_{2}\right)-B x_{2}}{m_{d} r_{d} x_{2}(\sin \alpha-n \cos \alpha)}} .
$$

As applied previously, to make the equation independent of $\alpha$, the derivative of (18) with respect to $\alpha$ has to be equal to 0 :

$$
\omega=\sqrt{\frac{n\left(M_{c} g x_{1}+m_{d} g x_{2}+A x_{2}\right)-B x_{2}}{m_{d} r_{d} x_{2} \sqrt{n^{2}+1}}} .
$$

Changing $k_{1}, k_{2}, b_{1}$, and $b_{2}$ changes the walking stability threshold of the washing machine; this change can be achieved using a semiactive suspension system. However, owing to the structural constraints of the semiactive suspension system used in this study, the only known knowledge about the suspension elements is the maximum force value that can be produced. Therefore, it can be said that $k_{1} \widehat{p}_{1}+$ $b_{1}\left(d \widehat{p}_{1} / d t\right)$ and $k_{2} \widehat{p}_{2}+b_{2}\left(d \widehat{p}_{2} / d t\right)$ are the only known values in (19), and it is assumed that these values are always equal to the maximum forces produced by suspension elements.

To change the maximum force values produced by the semiactive suspension elements for reducing vibrations, the angular position and angular acceleration values produced by the washing machine have to be interpreted. This can be done using the function $J$ that contains three-axis angular position $\left(\theta_{x}, \theta_{y}\right.$, and $\left.\theta_{z}\right)$ and three-axis angular acceleration $\left(\alpha_{x}, \alpha_{y}\right.$, and $\left.\alpha_{z}\right)$ values, with the angular axis acceleration values being squared owing to the importance of acceleration in the vibration behavior at high frequencies:

$$
J=\alpha_{x}^{2}+\alpha_{y}^{2}+\alpha_{z}^{2}+\theta_{x}+\theta_{y}+\theta_{z}
$$

According to the gradient of function $J$, the maximum force values produced by the suspension elements are chosen. When the gradient of function $J$ becomes higher, it indicates that the value of function $J$ will increase dramatically. Therefore, the vibrations will reach higher levels, to prevent which the suspension elements must apply lower force values to 
TABLE 1: Different maximum force values of suspension elements.

\begin{tabular}{lcc}
\hline$G$ & Left suspension $(N)$ & Right suspension $(N)$ \\
\hline $0-50$ & 120 & 112 \\
$50-100$ & 105 & 105 \\
$100-150$ & 95 & 90 \\
$150-200$ & 86 & 81 \\
$200-250$ & 76 & 72 \\
$250-300$ & 65 & 61 \\
$300-350$ & 55 & 51 \\
$350-400$ & 39 & 39 \\
$400-450$ & 28 & 28 \\
$450-500$ & 21 & 19 \\
$500-550$ & 18 & 13 \\
$550-600$ & 16 & 11 \\
\hline
\end{tabular}

the drum. Otherwise, the washing machine will show unstable vibration behavior:

$$
G=\frac{J(t)-J\left(t-T_{s}\right)}{T_{s}} .
$$

The intervals of function $G$ for different maximum force values of suspension elements are shown in Table 1.

Each interval corresponds to a different vibration level of the washing machine, and the maximum forces applied by the semiactive suspension elements change to reduce the vibrations so as to deal with the dynamic stability problem that may occur during the spinning cycle. While the gradient of $J$ increases, the maximum forces produced by the suspension elements decrease and vice versa. This process directly changes the effects of the suspension elements in the equation for the walking stability condition (19). Figure 2 shows the flowchart of the control algorithm used, for a better understanding.

\section{Experimental Studies}

Experiments were performed for the spinning cycle of a washing machine, increasing gradually from $200 \mathrm{rpm}$ to $900 \mathrm{rpm}$, which produces the most challenging vibration patterns from the viewpoint of dynamic stability. The maximum weight capacity of the washing machine is $7 \mathrm{~kg}$. The equation shown below is used as the first evaluation criterion (Papadopoulas and Papadimitriou's walking stability threshold adapted to a horizontal axis washing machine working with semiactive suspension system) for the success of the controlled semiactive suspension system. $M$ is the magnitude of $\omega$ at each sampling time and $N$ is the sum of samples:

$$
J_{\omega}=\frac{\sum_{i=0}^{N} M_{i}}{N} .
$$

The higher the value of function $J_{\omega}$, the more stable the washing machine. $\omega$ values have to be calculated for each sampling time to obtain $J_{\omega}$ values. As mentioned before, for each sampling time, vibration data are recorded, gradient of

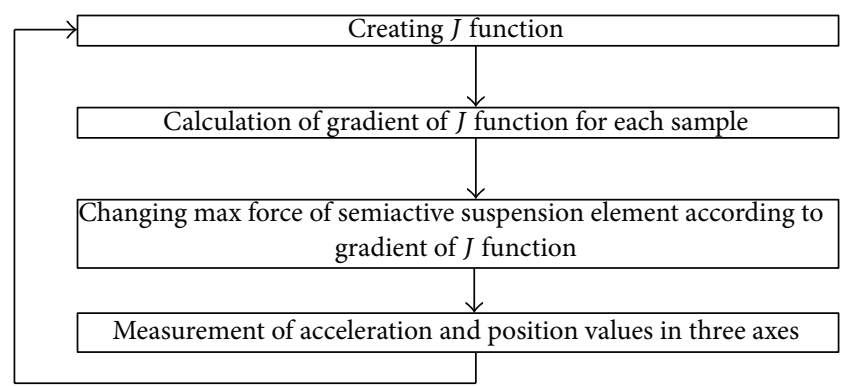

FIgURE 2: Flowchart of control algorithm.

$J$ function is calculated, and the maximum forces produced by suspension elements are changed to calculate $\omega$.

The second criterion is the arithmetic mean of the position and acceleration values in three axes. This criterion can also be used for investigating the changing rates of each position and acceleration parameters. Function $J_{v}$ is defined as follows:

$$
J_{v}=\frac{\sum_{i=0}^{N}\left|K_{i}\right|}{N},
$$

where $K$ is the magnitude of each signal (position or acceleration) at each sampling time and $N$ is the sum of samples. The lower the value of function $J_{v}$ is, the less oscillation it shows from its initial value 0 , indicating the success of the controller.

The process of changing the maximum forces of suspension elements can only be achieved at the same time owing to the structure of the motor drive module. A positive edge PWM signal is sent from the DAQ card to the motor drive module through a circuit including a $3 \mathrm{k}$ resistor and an NPN transistor, as shown in Figure 3. The connection between the resistor and the transistor is serial in nature.

A single PWM signal makes the suspension elements take only one step on the gradient line, and the number of PWM signals that have to be produced by the DAQ card is selected according to the gradient of function $J$. For instance, if the gradient of function $J$ is 3 at time $t$ and 6 at time $t+T_{s}$, three PWM signals have to be produced to change the maximum forces of the suspension elements. $T_{s}$ is selected as $300 \mathrm{~ms}$ for data acquisition and as $600 \mathrm{~ms}$ for control signal that drives step motors.

The MPU 6050 motion sensor is used in this study. It includes a 3-axis accelerometer and 3-axis gyroscope, and it has 16-bit analog-digital converters for digitizing the accelerometer and gyroscope outputs. The userprogrammable gyroscope scale is adjusted to $\pm 2000^{\circ} / \mathrm{s}$ and the user-programmable accelerometer scale to $\pm 16 \mathrm{~g}$. Even though the DAQ card provides $5 \mathrm{~V}$, the sensor has a voltage regulator for its working voltage of $3.3 \mathrm{~V}$. Communication with the data acquisition card is performed using the $\mathrm{I}^{2} \mathrm{C}$ protocol at $400 \mathrm{~Hz}$ from the SCL and SDA channels. The communication software is programmed in MATLAB using the $\mathrm{C}$ programming language. As mentioned in (8), walking instability first occurs around the front feet because they are subject to more force than the back feet. Thus, the sensor is 


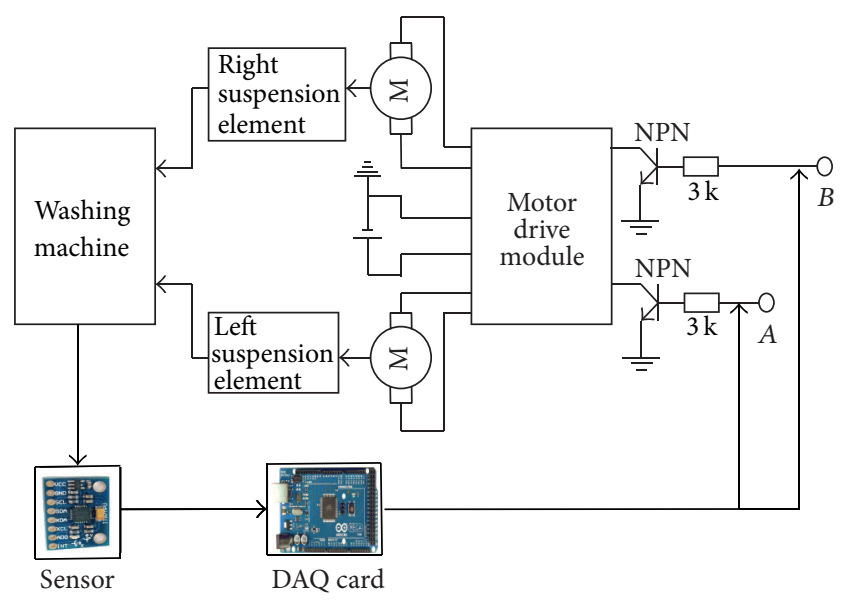

FIgURE 3: Motor drive module, sensor, and DAQ card connection.

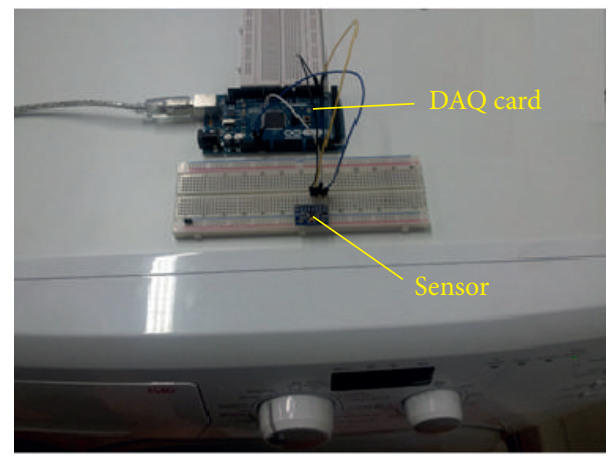

FIGURE 4: Sensor and DAQ card.

installed on the middle of the front-top panel of the washing machine, as shown in Figure 4.

The Arduino Mega 2560 DAQ card is used in this study. It includes an ATmega microcontroller, and communication between the PC and the DAQ card is performed using the serial communication protocol. A positive edge PWM signal is sent from the digital pin of the DAQ card after receiving sensor data. The communication software is programmed in MATLAB using the $C$ programming language.

The semiactive suspension system used in this study is shown in Figure 5. Inside the suspension elements, there is a cylindrical mass made of polyurethane. The data cable carries a signal from the motor drive module that drives the step motor. Depending on the type of signal, the bracelet starts to narrow or expand, causing a change in the elastic properties of the suspension element.

The experiments are performed in a semianechoic room, as shown in Figure 6. The sound power levels for uncontrolled and controlled suspension systems are measured according to ISO 3745.

The physical parameters of the washing machine are listed in Table 2. The trajectory of the passive spring elements is assumed to be $0.1 \sin (5 t)$ and $\beta_{1}, \beta_{2}, \beta_{3}$, and $\beta_{4}$ are assumed to be constant and equal to $55^{\circ}$. The friction coefficient between

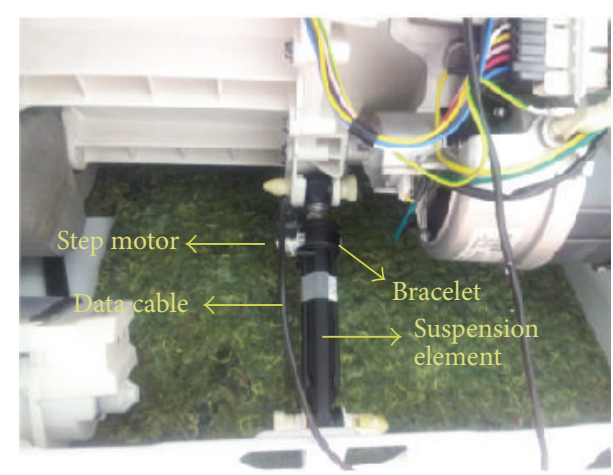

FIgURE 5: Semiactive suspension element.



FIGURE 6: Washing machine in anechoic chamber.

the feet and the ground is assumed to be constant and equal to 5 .

\section{Results and Discussion}

To show the success of the control method in damping vibrations, the measured position and acceleration values when the semiactive suspension system is uncontrolled (maximum force of left and right suspensions is 120 and 112, resp.) and controlled are shown in Figures 7-18.

As can be seen in Figure 7, for controlled system, variation between minimum and maximum values of $\theta_{x}$ is considerably low. Minimum value of $\theta_{x}$ is $-6^{\circ}$ and maximum value of $\theta_{x}$ is $+6^{\circ}$. On the other hand, for uncontrolled system shown in Figure 8 , the value of $\theta_{x}$ oscillates between $-60^{\circ}$ and $+60^{\circ}$, which means that $90 \%$ reduction of minimum and maximum values of vibration for $x$-axis is confirmed. Moreover, in Figure 7, it can be seen that the value of $\theta_{x}$ settles around $-1^{\circ}$; the reason of this situation is the noise that corrupts sensor data.

In Figure 9, the value of $\theta_{y}$ for controlled system settles around $0^{\circ}$ with a low value of steady-state error, which is described with $J_{v}$ in Table 3; variation between minimum and maximum values of $\theta_{y}$ is considerably low. Minimum value of $\theta_{y}$ is $-6^{\circ}$ and maximum value of $\theta_{y}$ is $+6^{\circ}$. For uncontrolled system as shown in Figure 10, the value of $\theta_{y}$ oscillates between $-60^{\circ}$ and $+40^{\circ}$, which means that minimum value 
TABLE 2: Physical parameters.

\begin{tabular}{cccccccccc}
\hline$D[\mathrm{~m}]$ & $x_{1}[\mathrm{~m}]$ & $x_{2}[\mathrm{~m}]$ & $M_{c}[\mathrm{~kg}]$ & $N$ & $k_{3}$ and $k_{4}[\mathrm{~N} / \mathrm{m}]$ & Trajectory $[\mathrm{m}]$ & $r_{d}[\mathrm{~m}]$ & $m_{d}[\mathrm{~kg}]$ & $\begin{array}{c}\beta_{1}, \beta_{2}, \beta_{3}, \text { and } \beta_{4} \\
{\left[{ }^{\circ}\right]}\end{array}$ \\
\hline 0.54 & 0.2 & 0.34 & 67 & 5 & 250 & $0.1 \operatorname{Sin}(5 t)$ & 0.2 & 10 & 55 \\
\hline
\end{tabular}

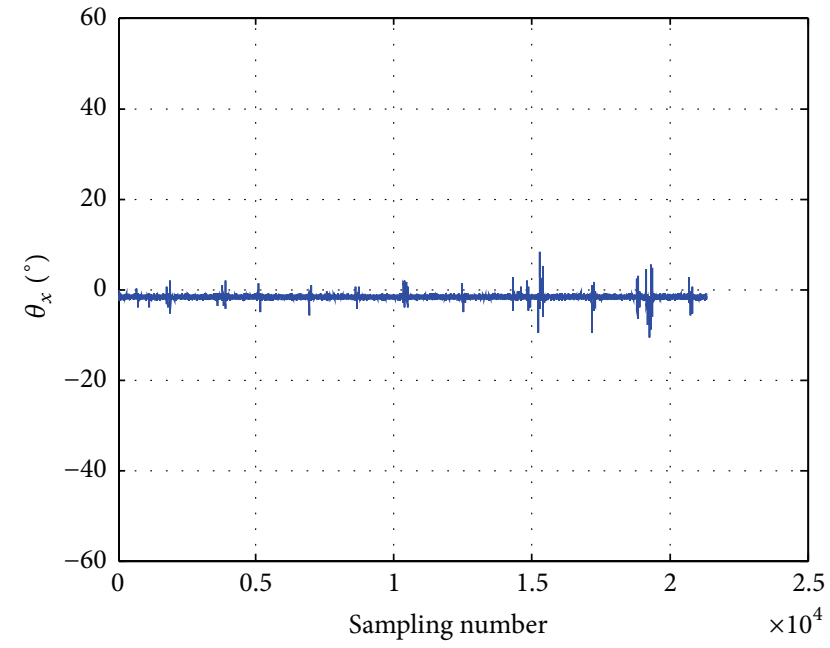

FIGURE 7: $\theta_{x}$ for controlled system.



FIGURE 8: $\theta_{x}$ for uncontrolled system.

of vibration in $y$-axis is reduced by $90 \%$, whereas maximum value of vibration in $y$-axis is reduced by $85 \%$.

In Figure 11, the value of $\theta_{z}$ for controlled system settles around $0^{\circ}$; peak values of $\theta_{z}$ are high with respect to steadystate error of $\theta_{z}$, whereas the value of $\theta_{z}$ settles around $+2^{\circ}$ for uncontrolled system as shown in Figure 12.

As can be seen from Figures 7-12, even though there are some peak values, vibrations in $x_{-}, y$-, and $z$-axes are considerably reduced for controlled system, especially for $\theta_{x}$ and $\theta_{y}$ parameters. Comparison for angular acceleration parameters is shown in Figures 13-18.

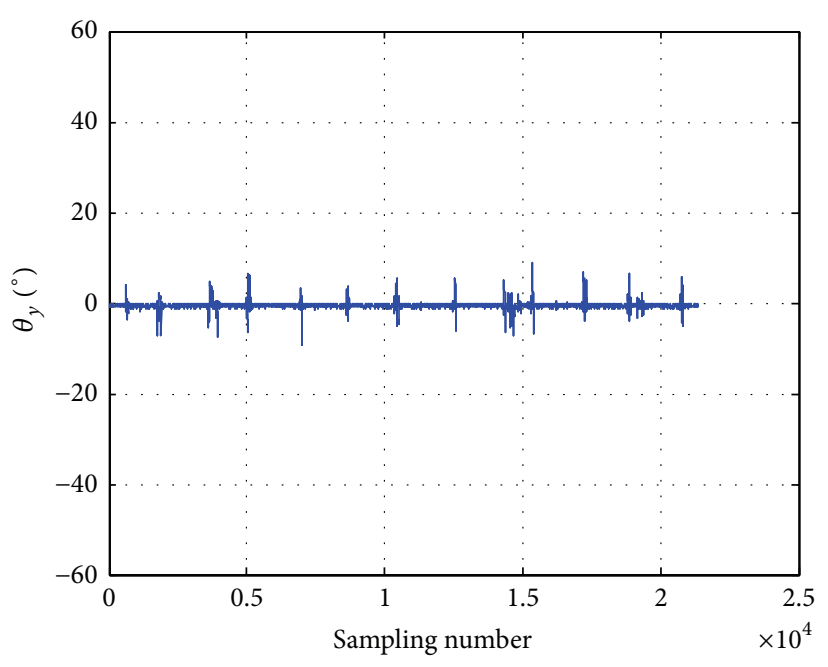

Figure 9: $\theta_{y}$ for controlled system.

TABLE 3: $J_{v}$ values of position and acceleration data for controlled and uncontrolled suspension system.

\begin{tabular}{lcccccc}
\hline$J_{v}$ values & $\theta_{x}\left[^{\circ}\right]$ & $\theta_{y}\left[{ }^{\circ}\right]$ & $\theta_{z}\left[{ }^{\circ}\right]$ & $\alpha_{x}\left[{ }^{\circ} / s^{2}\right]$ & $\alpha_{y}\left[{ }^{\circ} / s^{2}\right]$ & $\alpha_{z}\left[{ }^{\circ} / s^{2}\right]$ \\
\hline Controlled & 0.31 & 0.23 & 1.29 & 0.29 & 0.23 & 0.23 \\
Uncontrolled & 21.13 & 19.36 & 2.11 & 0.53 & 0.95 & 0.57 \\
\hline
\end{tabular}

TABLE 4: Improvement percentage of $J_{v}$ values for position and acceleration data compared with their uncontrolled values.

\begin{tabular}{lccccc}
\hline$\theta_{x}$ & $\theta_{y}$ & $\theta_{z}$ & $\alpha_{x}$ & $\alpha_{y}$ & $\alpha_{z}$ \\
\hline $98.53 \%$ & $98.81 \%$ & $38.86 \%$ & $45.28 \%$ & $75.78 \%$ & $59.64 \%$ \\
\hline
\end{tabular}

In Figure 13, the value of $\alpha_{x}$ for controlled system settles around $-0.4^{\circ}$; peak values of $\alpha_{x}$ are high with respect to steady-state error of $\alpha_{x}$ and increase through the end of washing operation. For uncontrolled system shown in Figure 14, it can be seen that the value of $\alpha_{x}$ oscillates between $-1^{\circ}$ and $+0.7^{\circ}$.

In Figure 15, the value of $\alpha_{y}$ for controlled system settles around $0^{\circ}$; peak values of $\alpha_{y}$ are the highest ones among other angular acceleration parameters shown in Figures 13 and 17. However, this situation does not corrupt improvement percentage of $J_{v}$ as can be seen in Table 4. For uncontrolled system shown in Figure 16, it can be seen that the value of $\alpha_{x}$ oscillates between $-1^{\circ}$ and $+0.7^{\circ}$ and there are minimum and maximum peak values around $-4^{\circ}$ and $+4^{\circ}$, respectively.

In Figure 17, the value of $\alpha_{z}$ for controlled system settles around $0^{\circ}$ and varies between $-2^{\circ}$ and $+2.5^{\circ}$. For uncontrolled system shown in Figure $18, \alpha_{z}$ settles around $0^{\circ}$ with high frequency change. 


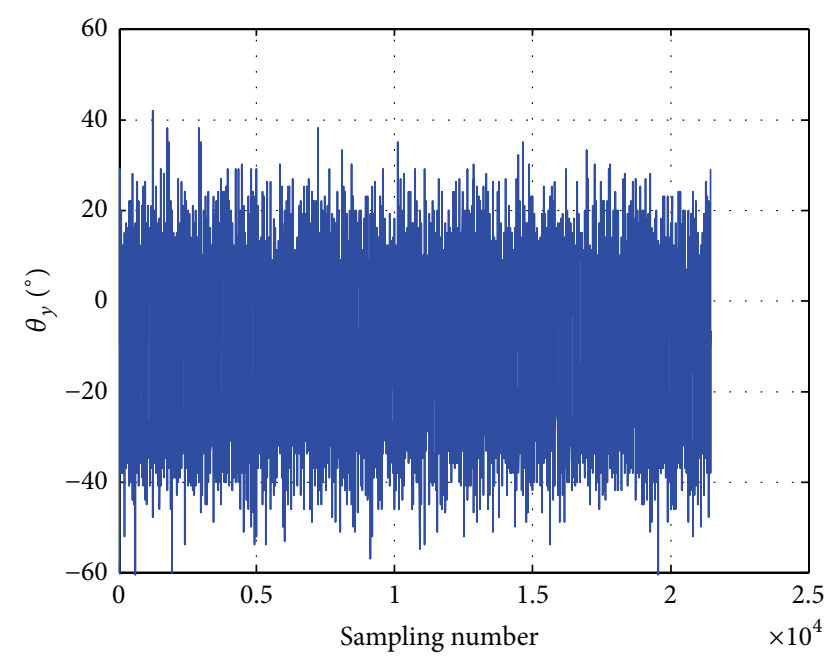

FIgURE 10: $\theta_{y}$ for uncontrolled system.

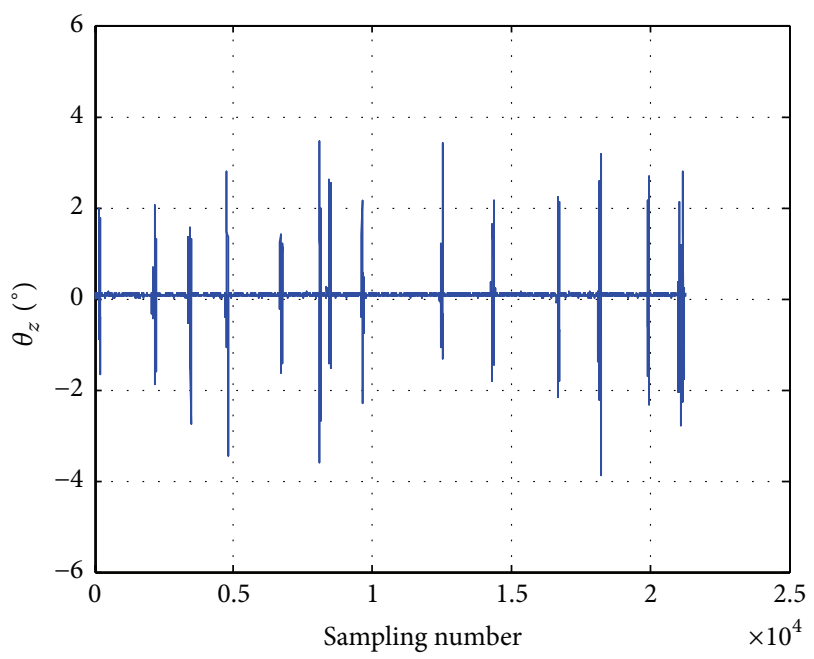

FIGURE 11: $\theta_{z}$ for controlled system.

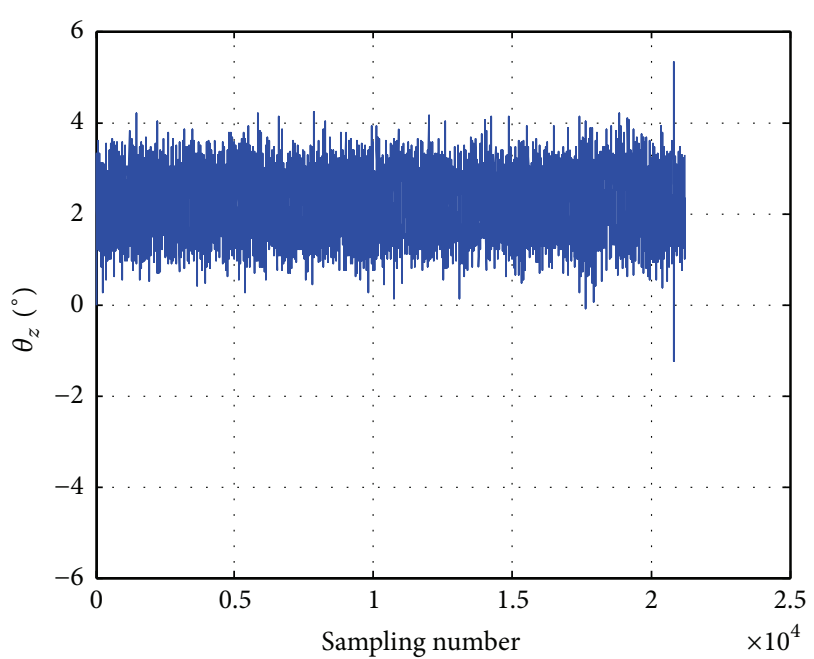

FIGURE 12: $\theta_{z}$ for uncontrolled system.

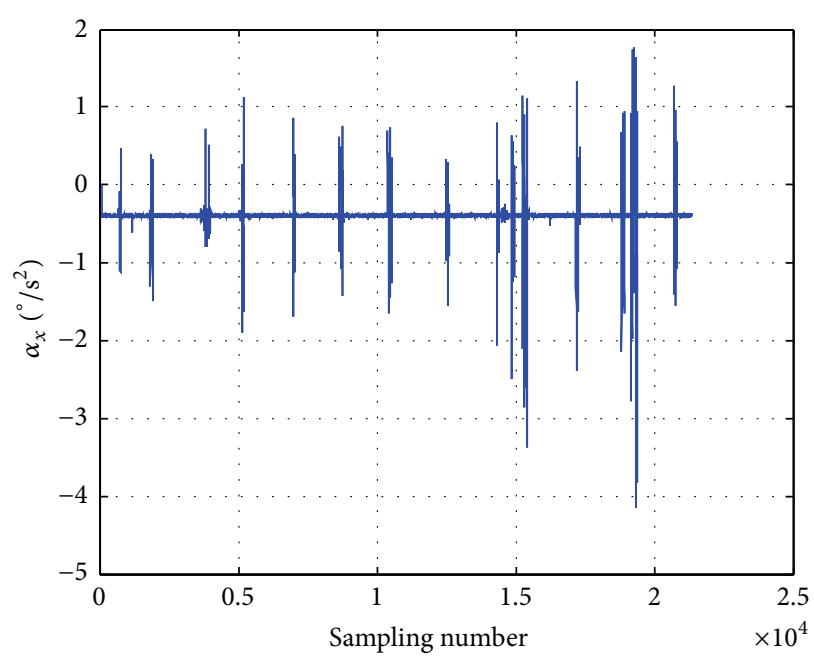

FIGURE 13: $\alpha_{x}$ for controlled system.



FIGURE 14: $\alpha_{x}$ for uncontrolled system.

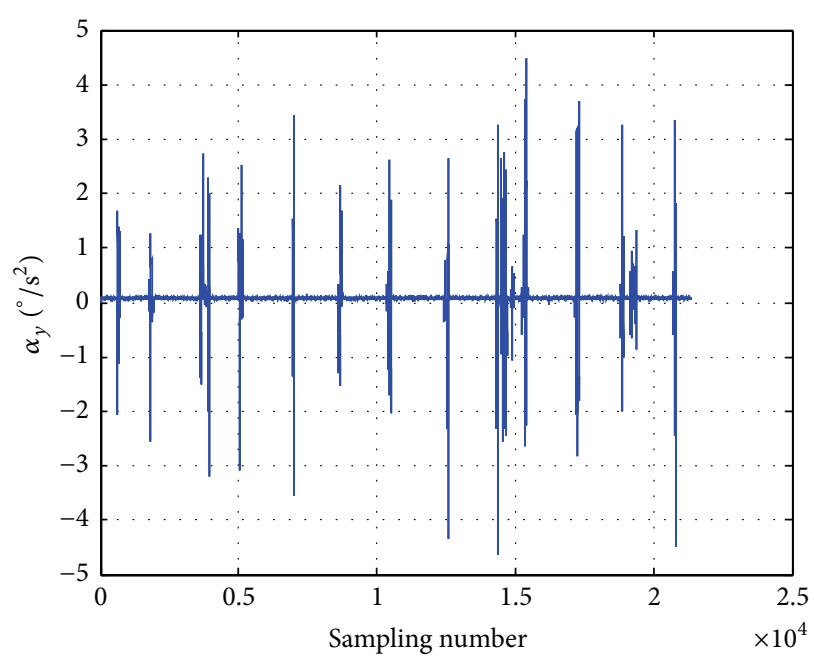

FIGURE 15: $\alpha_{y}$ for controlled system. 


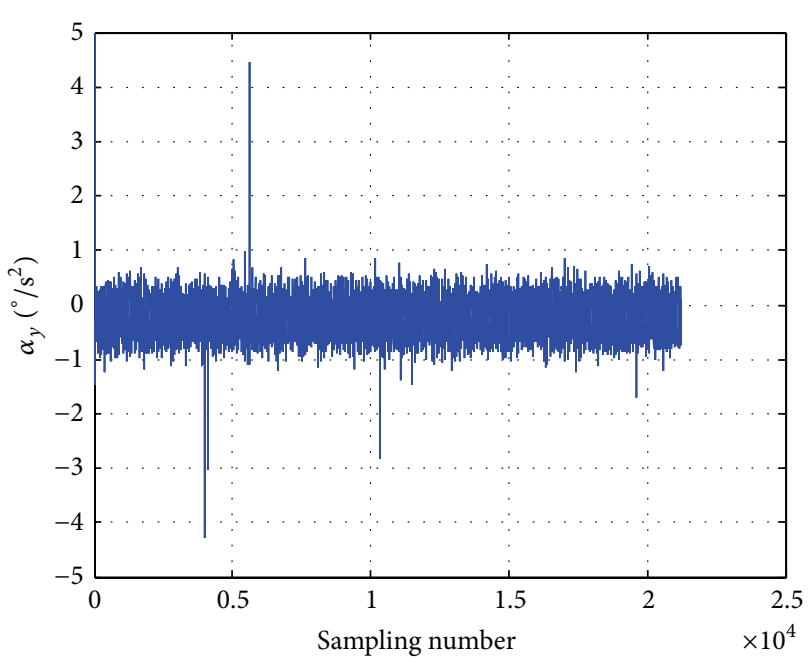

FIGURE 16: $\alpha_{y}$ for uncontrolled system.

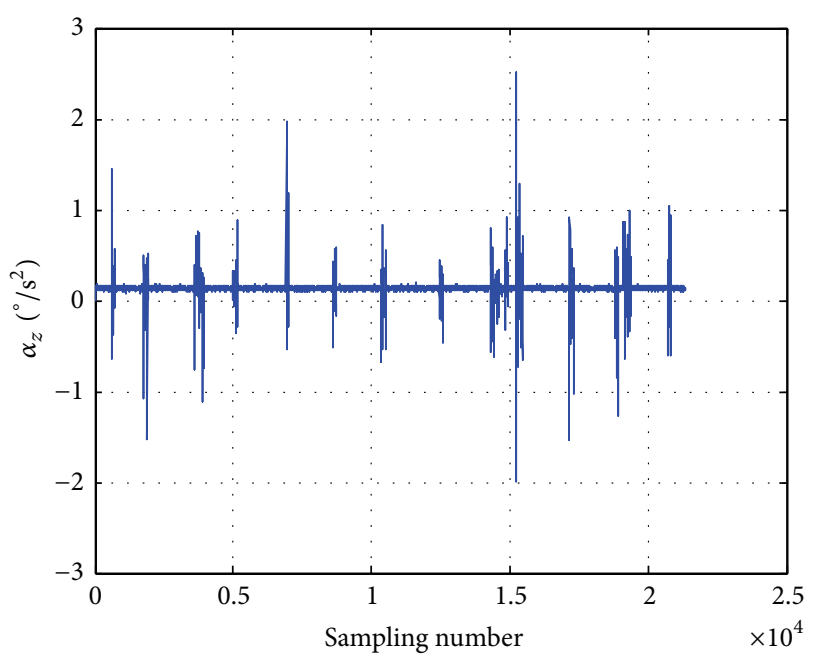

FIGURE 17: $\alpha_{z}$ for controlled system.

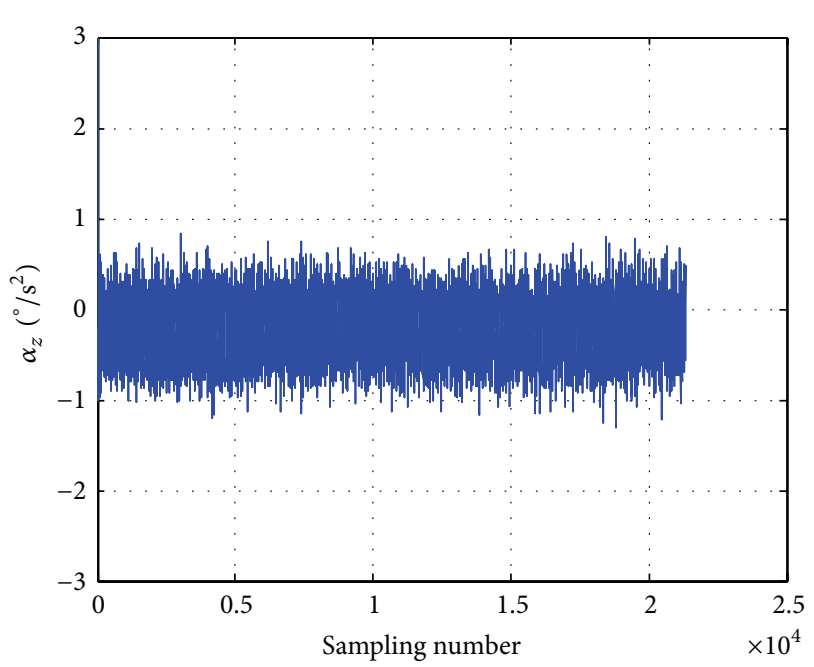

FIGURE 18: $\alpha_{z}$ for uncontrolled system.

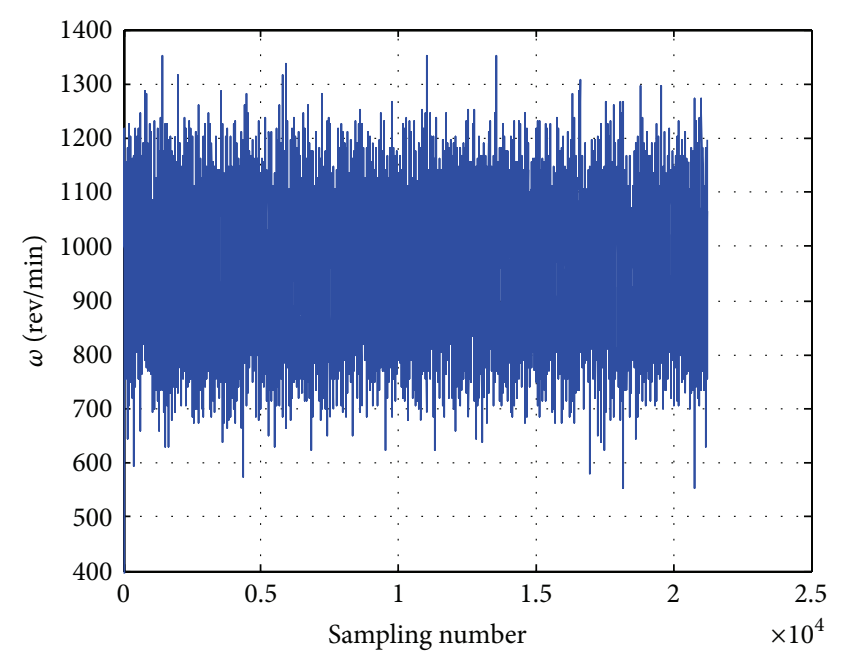

FIGURE 19: $\omega$ for controlled suspension system.

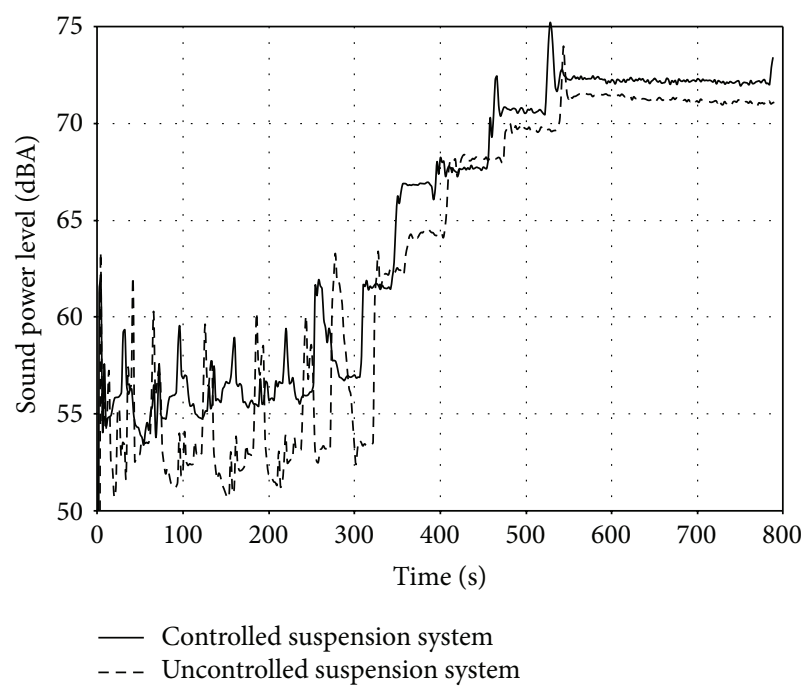

FIgURE 20: Measured sound power levels for controlled and uncontrolled suspension systems.

As can be seen from Figures 13, 15, and 17, angular acceleration parameters for controlled system have peaks, just as angular position parameters shown in Figures 7, 9, and 11; however, in this case, the peak values reach beyond the boundaries of uncontrolled system's angular acceleration outputs. The main reason of the existence of these peak values is that, due to hardware constraints, the sampling time $T_{s}$ is adjusted to $300 \mathrm{~ms}$ for data acquisition while it is adjusted to $600 \mathrm{~ms}$ for control signal that drives step motors. When the gradient of function $J$ drastically changes (and the system becomes prone to be unstable), the control signal cannot quickly affect the system because of time delay between data acquisition and control signal.

The $J_{v}$ values of each position and acceleration data for a controlled and an uncontrolled semiactive suspension system are listed in Table 3.

Table 4 suggests that the controller mostly succeeds in terms of the position $\theta_{y}$ and acceleration $\alpha_{y}$. 
The change in $\omega$ over time is shown in Figure 19; $J_{\omega}$ is calculated as $1080.2 \mathrm{rpm}$.

Sound power levels of the washing machine for controlled and uncontrolled cases have also been measured according to ISO 3745 in a semianechoic room as shown in Figure 6 and the measurement results are presented in Figure 20.

Figure 20 shows that at the low spin speeds the sound power level is more stable when the semiactive suspension system is controlled; in comparison, the uncontrolled system shows more oscillatory behavior for the first spinning cycles. At the highest final spin speed, $900 \mathrm{rpm}$, Figure 20 shows that there is no major difference between the measured sound power levels.

In Figure 20, it can be seen that the value of sound power changes between $50 \mathrm{dBA}$ and $63 \mathrm{dBA}$ when the system is uncontrolled and the value of sound power changes between $54 \mathrm{dBA}$ and $62 \mathrm{dBA}$ when the system is controlled for the first spinning cycles.

\section{Conclusions}

This study proves that low-cost equipment including an MPU 6050 sensor, an Arduino Mega 2560 used as data acquisition card, and a semiactive suspension system working based on maximum force adjustment can be used to solve the walking instability problem of washing machines. When the system is controlled, angular position values in $x$-, $y$-, and $z$-axes settle around $0^{\circ}$ with low steady-state errors, which means that they are considerably reduced. Even though angular acceleration values have peaks during the operation, they have not any major effects on walking and dynamic stability.

Finally, the measured sound power levels prove that a washing machine with a controlled semiactive suspension system shows more stable sound performance than an uncontrolled one for the spinning cycle. Furthermore, even though there is an enormous difference between the controlled and the uncontrolled semiactive suspension systems from the viewpoint of vibration damping, the final sound power levels of the washing machine for both conditions are almost the same (72 dBA and $73 \mathrm{dBA})$, suggesting that there is no linear relation between vibrations causing walking instability and sound power levels produced by the washing machine in this case.

\section{Conflict of Interests}

The authors declare that there is no conflict of interests regarding the publication of this paper.

\section{References}

[1] D. C. Conrad and W. Soedel, "On the problem of oscillatory walk of automatic washing machines," Journal of Sound and Vibration, vol. 188, no. 3, pp. 301-314, 1995.

[2] D. C. Conrad, The fundamentals of automatic washing machine design based upon dynamic constraints [Ph.D. thesis], Purdue University, Ann Arbor, Mich, USA, 1994.

[3] T. Nygårds and V. Berbyuk, "Optimization of washing machine kinematics, dynamics, and stability during spinning using a multistep approach," Optimization and Engineering, vol. 15, no. 2, pp. 401-442, 2014.

[4] A. Ashfak, K. K. A. Rasheed, and J. A. Jaleel, "Modeling, simulation and experimental validation of Magneto-Rheological damper," in Proceedings of the International Conference on Advanced Nanomaterials and Emerging Engineering Technologies (ICANMEET '13), pp. 267-274, July 2013.

[5] C. Spelta, F. Previdi, S. M. Savaresi, G. Fraternale, and N. Gaudiano, "Control of magnetorheological dampers for vibration reduction in a washing machine," Mechatronics, vol. 19, no. 3, pp. 410-421, 2009.

[6] O. S. Türkay, B. Kiray, A. K. Tugcu, and İ. T. Sümer, "Formulation and implementation of parametric optimisation of a washing machine suspension system," Mechanical Systems and Signal Processing, vol. 9, no. 4, pp. 359-377, 1995.

[7] O. S. Türkay, I. T. Sümer, A. K. Tuğcu, and B. Kiray, "Modeling and experimental assessment of suspension dynamics of a horizontal-axis washing machine," Journal of Vibration and Acoustics, vol. 120, no. 2, pp. 534-543, 1998.

[8] P. Boyraz and M. Gündüz, "Dynamic modeling of a horizontal washing machine and optimization of vibration characteristics using Genetic Algorithms," Mechatronics, vol. 23, no. 6, pp. 581593, 2013.

[9] M. E. Öztürk and H. Erol, "On the dynamics of a washing machine with flexible components," Noise Control Engineering Journal, vol. 58, no. 6, pp. 572-590, 2010.

[10] E. Papadopoulos and I. Papadimitriou, "Modeling, design and control of a portable washing machine during the spinning cycle," in Proceedings of the IEEE/ASME International Conference on Advanced Intelligent Mechatronics, pp. 899-904, July 2001.

[11] M. Kalkat, "Experimentally vibration and noise analysis of two types of washing machines with a proposed neural network predictor," Measurement, vol. 47, no. 1, pp. 184-192, 2014. 

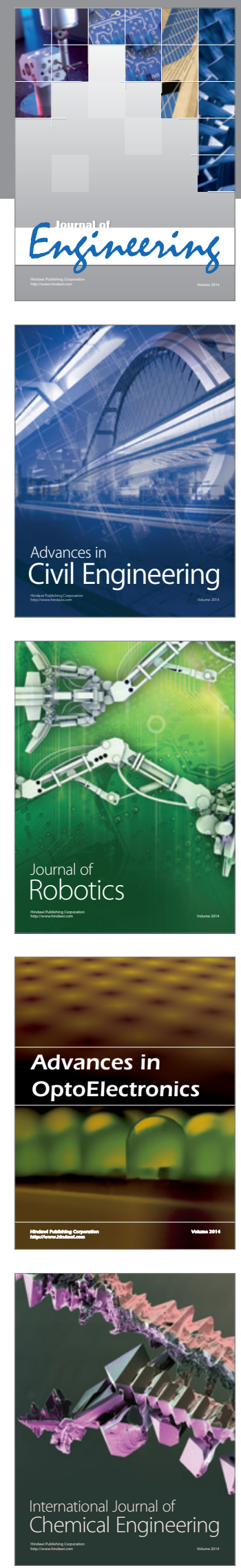



The Scientific World Journal
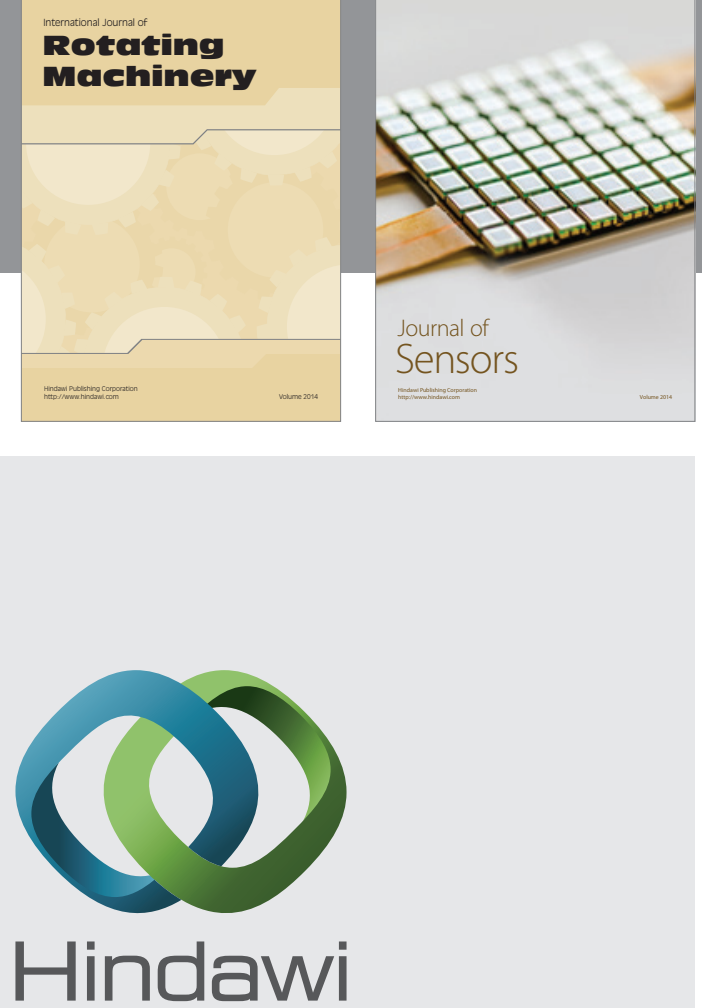

Submit your manuscripts at http://www.hindawi.com
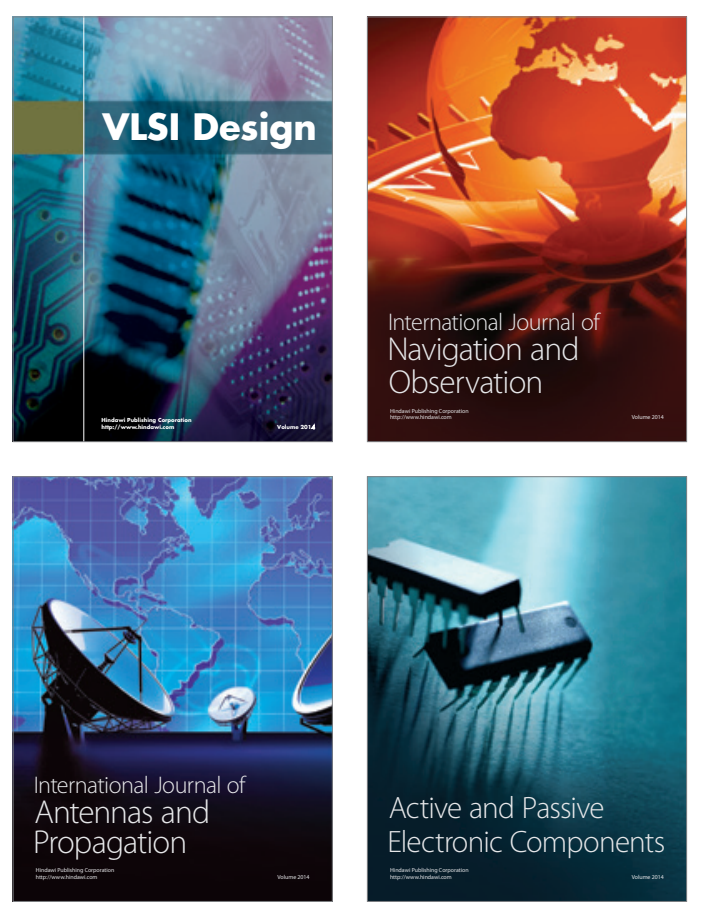
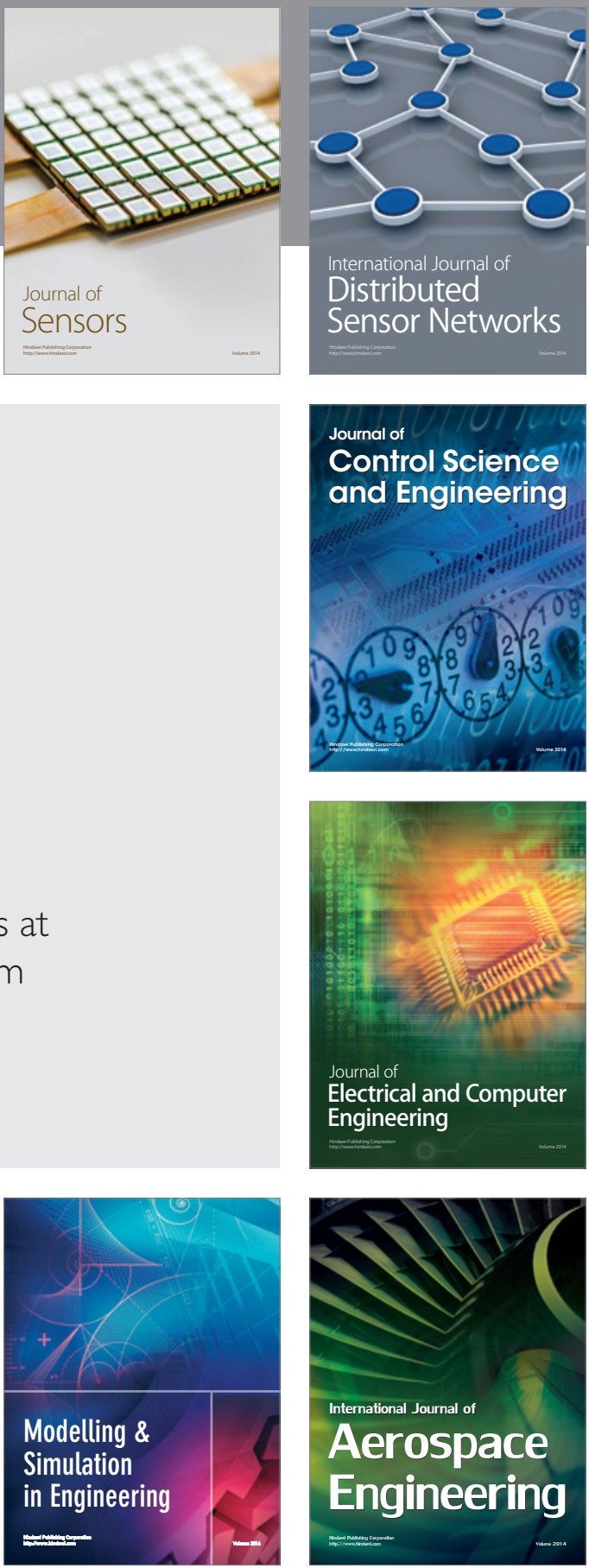

Journal of

Control Science

and Engineering
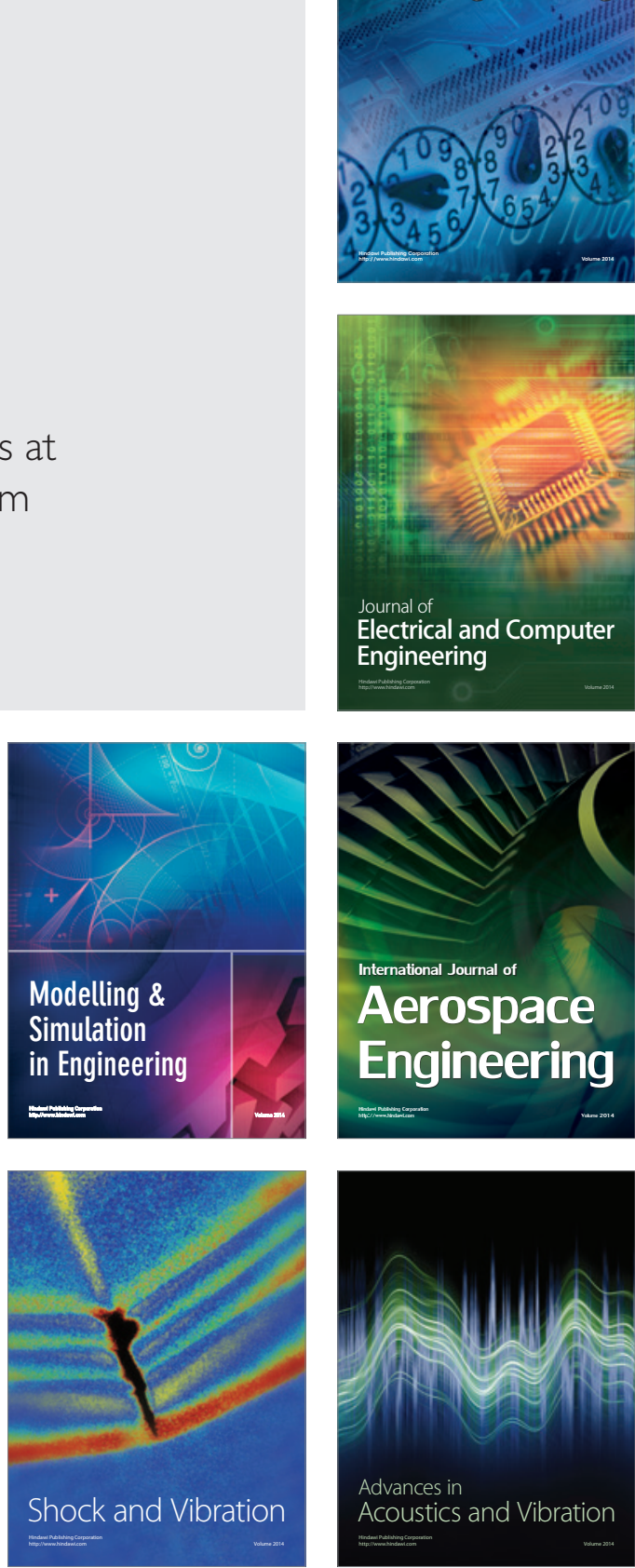